\title{
Role of clathrin in the inmune synapse formation
}

C. Calabia-Linares*, M. J. Alfonso-Pérez*, N. Martín-Cofreces*, M. Pérez-Martínez*, C. AguadoBallano**, F. R. Urbano-Olmos**, C. O. Sánchez-Sorzano***, F. Sánchez-Madrid*, E. Veiga*

*Immunology Department, Hospital Universitario de la Princesa, Universidad Autónoma de Madrid. 28006 Madrid, Spain

**Laboratorio de Microscopía Electrónica de Transmisión, Facultad de Medicina, Universidad Autónoma de Madrid. 28029 Madrid, Spain

***Centro Nacional de Biotecnología CSIC, Unidad de Biocomputación, Cantoblanco, 28049 Madrid, Spain.

To become activated, T-cells must establish cell-cell contact with antigen-presenting cells (APCs). This contact known as the immune synapse (IS) involves the interaction of the TCR (T-cell receptor) with antigen-containing major histocompatibility complex (MHC-I or MHC-II) from the APC and other T-cell-APC molecular interactions. TCR activation in the IS drives major morphological and functional changes in T-cells, including massive actin rearrangements. This Actin polymerization is absolutely necessary for productive IS maturation $[1,2]$. Many components of the signalling pathways have been identified largely by advances in understanding actin biology in nonhaematopoietic cells. In many aspects, the cytoskeletal dynamics triggered by TCR activation in the IS resembles events occurring in the other systems i.e. lamellipodium of a migrating cell.

Nevertheless, the ultimate link between the TCR activation and massive actin polymerization is unclear. Recently it have been proposed that clathrin may serve as a platform for actin polymerization $[3,4]$, thus we are exploring the role that clathrin may play in the IS formation. We are studying the dynamics of clathrin at the IS, as well as the IS ultra-structure combining low resolution imaging techniques (i.e fluorescence, confocal microscopy) with high resolution microscopy (i.e. Electron, and X-ray microscopy). It is in our aim to integrate the 3D spatial information provided by each microscopy at different resolutions (4D) and at different time stages of the synapse $(5 \mathrm{D})$.

\section{References}

[1] D.D. Billadeau et al., Traffic 7 (2006) 1451.

[2] D.D. Billadeau et al., Nat. Rev. Immunol. 7 (2007) 131.

[3] E. Veiga et al., Nat. Cell Biol. 7 (2005) 894.

[4] E. Veiga et al., Cell Host and Microbe 2 (2007) 340. 


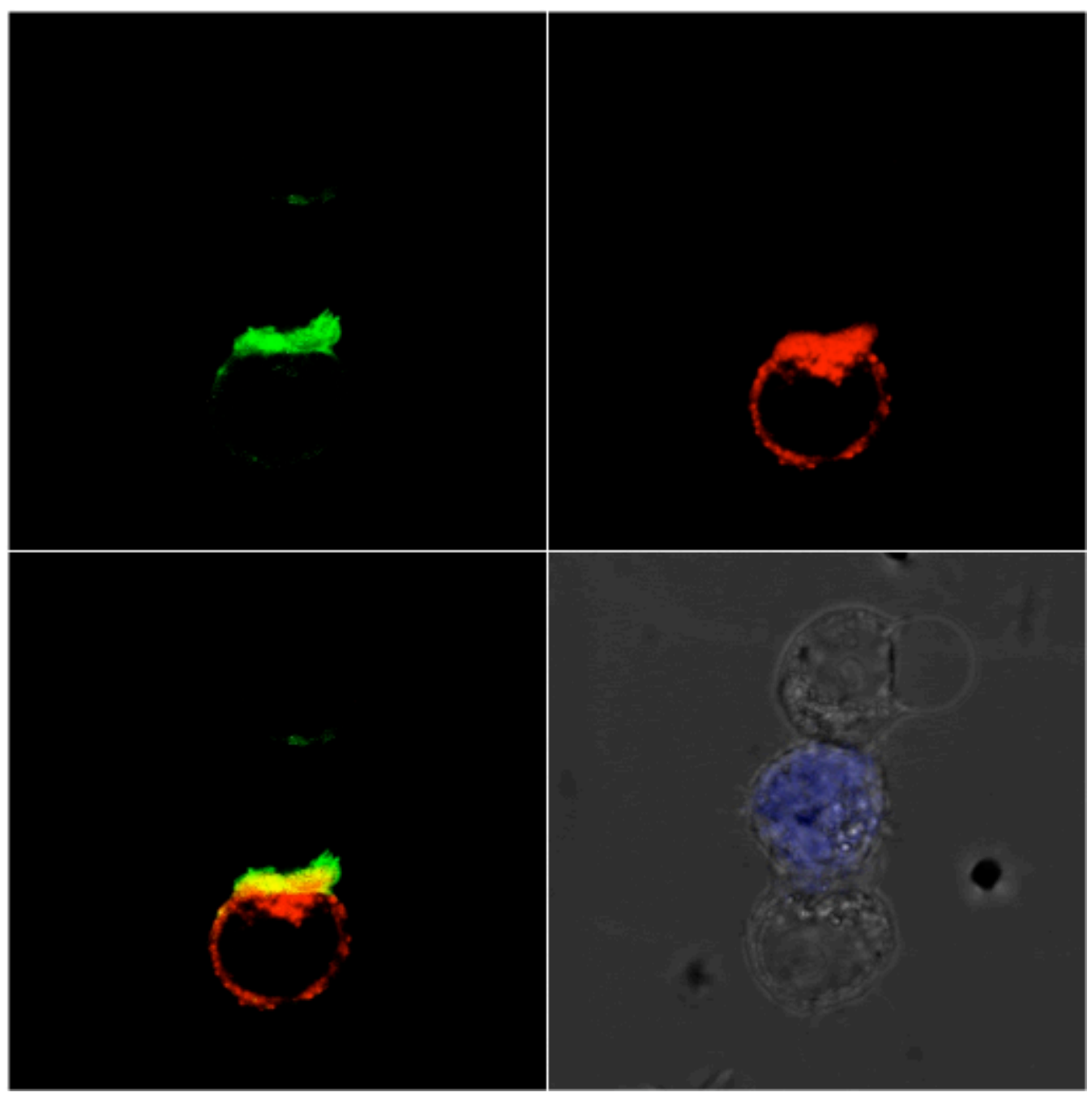

FIG. 1. The figure shows a T-cell expressing actin-GFP (green) and clatrhin-Tomato (red) forming an immune synapse with an antigen presenting cell (APC) in blue in the last image. 